\title{
ERRATUM TO "RATIONAL SMOOTHNESS AND FIXED POINTS OF TORUS ACTIONS"
}

\author{
M. BRION \\ Institut Fourier, B. P. 74 \\ F-38402 Saint-Martin d'Hères, France \\ Michel.Brion@ujf-grenoble.fr
}

As pointed out by T. A. Springer, the first assertion of the lemma in Section 3.2 is incorrect. This lemma concerns orbit closures of $B \times B^{-}$in $X$, where $B$ and $B^{-}$ are opposite Borel subgroups of a connected reductive group $G$, and $X$ is a regular $G \times G$-equivariant completion of $G$.

Specifically, with the notation of the lemma, the closure of the $\left(B \times B^{-}\right)$-orbit $(B \times$ $\left.B^{-}\right)(w, \tau) y$ in its $(G \times G)$-orbit $(G \times G) y$ contains all orbits $\left(B \times B^{-}\right)\left(w^{\prime}, \tau^{\prime}\right) y$, where $w^{\prime}, \tau^{\prime} \in W$ satisfy $w^{\prime} \geq w$ and $\tau^{\prime} w_{0, L} \geq \tau w_{0, L}$. But this closure may contain additional $\left(B \times B^{-}\right)$-orbits as well, contrary to the first assertion of the lemma. The argument of proof actually shows that the closure

$$
\overline{\left(B \times B^{-}\right)(w, \tau) y} \cap(G \times G) y
$$

is the union of the $\left(B \times B^{-}\right)$-invariant subsets

$$
\left(B \times B^{-}\right)\left(w^{\prime}, \tau^{\prime} w_{0, L}\right)\left(B^{-} \times B\right)\left(1, w_{0, L}\right) y
$$

for all $\left(w^{\prime}, \tau^{\prime}\right)$ as above.

Since this lemma is only used (in the proof of Theorem 3.3) to construct certain $\left(B \times B^{-}\right)$-orbits in the closure $\overline{B w_{0} B^{-}}=\bar{B} w_{0}$, this error does not affect the other results of the paper.

Several combinatorial descriptions of all inclusion relations between $B \times B$-orbit closures in $X$ are to be found in Section 2 of the preprint "Intersection cohomology of $B \times B$ orbit closures in group compactifications" by T. A. Springer, to appear in the Journal of Algebra. This preprint is available at the URL: http://www.math.uu.nl/people/vdkallen.

Received August 18, 2001. 